\title{
Advective flow in a sludge floc
}

\author{
C.P. Chu, D.J. Lee* \\ Chemical Engineering Department, National Taiwan University, Taipei 10617, Taiwan
}

Received 8 February 2004; accepted 22 April 2004

Available online 28 May 2004

\begin{abstract}
The interior of sludge floc is highly heterogeneous, while the large pores in the floc control the advective flow. This work for the first time numerically details fluid flow and mass transfer processes in pores of activated sludge floc. The dimensionless permeabilities and mass dispersion coefficients were contoured against pore size ratio and the floc Reynolds number. With a pore size less than $20 \%$ of the floc size, the commonly adopted homogeneous model overestimates the floc permeability, and pore velocity is less than $2 \%$ of the bulk velocity. This is particularly true for flocs with low porosity. Although the convective flux is low, the dispersive mass transfer rate can be much higher than the diffusional rate, attributable to the strong Taylor dispersion effect. The three-dimensional pore structures in waste activated-sludge floc were identified using confocal laser scanning microscope (CLSM) images. Large pores were used to numerically estimate the permeability and dispersion coefficient for these pores. The permeability and the dispersion coefficient of the tortuous pores can be one order of magnitude lower than those for the equivalent straight pores. Besides the dispersion effect, the pore tortuosity appeared as the most important geometrical factor retarding the advective flow in the sludge pores. In addition, the small side pores connected to the large pore had only a mild effect on the flow process, and can be neglected in analysis.
\end{abstract}

(c) 2004 Elsevier Inc. All rights reserved.

Keywords: Floc model; Pore; Permeability; Dispersion coefficient; CLSM; Tortuosity

\section{Introduction}

Knowledge of the hydrodynamic drag force exerted on a floc is necessary for predicting its motion [1]. The hydrodynamic force exerted on a floc of diameter $d_{\mathrm{f}}(\mathrm{m})$ moving at a steady velocity of $U(\mathrm{~m} / \mathrm{s})$ is

$F_{\mathrm{S}}=\left(\frac{\pi}{4} d_{\mathrm{f}}^{2}\right)\left(\frac{1}{2} \rho U^{2}\right) \Omega C_{\mathrm{D}}$,

where $C_{\mathrm{D}}$ and $\rho$ are the drag coefficient (-) and fluid density $\left(\mathrm{kg} / \mathrm{m}^{3}\right)$, respectively. Meanwhile, the correction factor $\Omega$ (一) takes account of the advection flow through the floc's interior, which is unity for an impermeable floc, and less than unity for a porous floc. The hydrodynamic permeability of a hypothetical "point" in a porous medium, $k\left(\mathrm{~m}^{2}\right)$, can be defined as

$k=-\frac{\mu}{\nabla p} \vec{U}_{\text {rel }}$

\footnotetext{
* Corresponding author. Fax: +886-2-2362-3040. E-mail address: djlee@ccms.ntu.edu.tw (D.J. Lee).
}

where $\vec{U}_{\text {rel }}(\mathrm{m} / \mathrm{s})$ is the relative velocity of liquid and the solid phases, $p(\mathrm{~Pa})$, the local pressure, and $\mu(\mathrm{Pas})$ the liquid phase viscosity. Hence, the permeability controls the extent of the advection flow. Experimental efforts were devoted to estimating the floc permeability using hydrodynamic tracking techniques [2-7]. In most tests the advection flow through the floc interior is noted. Recent related reviews can be found in $[8,9]$. Conventional models assumed that molecular diffusion controlled the intrafloc transport processes. Logan and Hunt [10,11] pioneered the discussions on the possible role of advective flow on the intrafloc transport processes.

Floc interior is heterogeneous in nature [12-14]. Large pores can exist in the sludge flocs $[15,16]$. Moisture tends to flow through the path of least resistance; hence, water would be unlikely to flow into the tiny pores in the microflocs, but likely to flow through the large pores among the microflocs. Permeability measured thereby is mainly attributed to the structures of the large pores [17]. Conventionally, theoretical permeability models assume a homogeneous floc interior [18-22]. The revised, fractal-like floc model has been used 
to explore the hydrodynamic behavior and the coagulation characteristics of floc [23-26].

Wu et al. [27] adopted a very simple heterogeneous floc model, considering the floc as a cube of size $d_{\mathrm{f}}$ that consists of $N$ parallel capillary tubes of diameter $d_{\mathrm{p}}$ and length $L$. The remaining portion of the cube is impermeable. Considering the fluid flow in this simple model, $\mathrm{Wu}$ et al. noted that most fluid is flowing through the biggest pore within the floc, whence the porosity calculated based on homogeneous models would be close to unity regardless of the real (intrinsic) porosity. Discussions of the advective process should be based on information on the biggest pores in the sludge floc. In biological flocs, however, some authors commented that the advective flow may not be so significant as expected, since intrafloc tunnels can be clogged by excellular polymers (ECPs) yielded by cells [7,28,29]. Ploug [30] demonstrated that the interfacial oxygen transfer rate was not the controlling factor in the intra-aggregate transport process. Woodfield and Bickert [31] proposed a fractal model considering the floc as an aggregate made up of a swarm of permeable spheres.

We numerically explored the flow process and solute mass transfer in a single pore of a sludge floc. A model floc with a straight pore located at its centerline was used to demonstrate the effects of pore size (porosity) and floc Reynolds number on the permeability and dispersion coefficients of the advective flow. Then the structural information from real pores in sludge floc was adapted to construct the $3 \mathrm{D}$ pore model. Effects of pore tortuosity were analyzed. If certain pores were clogged, the resistances of fluid flow or dispersive mass transfer within a floc would be further retarded than those presented herein.

\section{Flow in straight pores}

\subsection{The flow field}

\subsubsection{Model and governing equations}

We consider a sphere of diameter $d_{\mathrm{f}}$ with one straight pore of diameter $d_{p}$ passing through its center. The porosity of the sphere is thereby defined as the (void space of the sphere)/(solid space of the sphere). The sphere is moving at a steady speed of $U$ through an infinite, quiescent Newtonian fluid of viscosity $\mu$ and density $\rho$. The approaching fluid of velocity $U$ moves parallel to the straight pore. For the sake of easy definition of boundary conditions, an equivalent problem is considered: the sphere is fixed at the centerline while the surrounding fluid is flowing at a uniform speed of $\vec{U}$ from infinity toward the fixed sphere [32].

The following steady-state Navier-Stokes equations control the fluid flow field,

$$
\left(\vec{u}_{\mathrm{f}}^{*} \cdot \nabla^{*}\right) \vec{u}_{\mathrm{f}}^{*}+E u \nabla^{*} p^{*}=\frac{1}{R e_{\mathrm{f}}} \nabla^{* 2} \vec{u}_{\mathrm{f}}^{*}
$$

where $\vec{u}_{\mathrm{f}}$ is the fluid velocity,

$\nabla^{*}=\frac{\nabla}{d_{\mathrm{f}}}, \quad \vec{u}_{\mathrm{f}}^{*}=\frac{\vec{u}_{\mathrm{f}}}{U}, \quad E u=\frac{p_{0}}{\rho U^{2}}, \quad R e_{\mathrm{f}}=\frac{\rho U d_{\mathrm{f}}}{\mu}$,

and $p^{*}=p / p_{0}$. The first and second terms of the left hand side of Eq. (3) correspond to the inertia and pressure effects, respectively. Meanwhile, its right hand side refers to the viscous effect. The boundary conditions are as follows:

$\vec{u}_{\mathrm{f}}^{*}=\overrightarrow{1} \quad$ at $r^{*} \rightarrow \infty$,

$\frac{\partial \vec{u}_{\mathrm{f}}^{*}}{\partial r^{*}}=\overrightarrow{0} \quad$ at $r^{*}=0$,

$\vec{u}_{\mathrm{f}}^{*}=\overrightarrow{0} \quad$ at the sphere and pore surface.

Equation (4a) states that the surrounding fluid is moving at a constant speed when away from the sphere. Equation (4b) addresses the symmetric line. Equation (4c) is the no-slip boundary condition. The entry and exit of the pore are open to the surrounding fluid field.

The governing equation, Eq. (3), is solved with the associated boundary conditions Eqs. (4a)-(4c) by the computational fluid dynamics program FIDAP 7.6 (FDI Inc., USA). This software employs a finite element scheme with bilinear, four-node quadrilateral elements. Since the flow process is symmetrical along the $r=0$ axis, the axis-symmetry flow is employed. We consider a domain "tube" for the fluid field of length $L$ and radius $R$. The numbers of elements in the fluid side is about 10,000 . Since it is the fluid field within and around the sphere that is of major concern, more elements are allocated near the sphere for better accuracy.

The numerical solution cannot be accomplished in an infinite domain such as Eq. (4a). In numerical practice, the ratio $R / d_{\mathrm{f}}$ would not influence the calculation results if it exceeded 7.5. That is, the domain tube can be used to represent an infinite flow field. In this study, the $L / d_{\mathrm{f}}$ and $R / d_{\mathrm{f}}$ are fixed at 150 and 30 , respectively. The calculations are performed at a maximum relative error of $0.1 \%$. With the fluid velocity field within and around the sphere the hydrodynamic drag force can be numerically evaluated for $d_{\mathrm{p}} / d_{\mathrm{f}}=0.5372(\varepsilon=0.40)$ and $0.9765(\varepsilon=0.99)$, respectively, using the postprocessing program FIPOST.

\subsubsection{Flow pattern}

For the sphere with $\varepsilon=0.40$ (Fig. 1), the originally flatshaped flow field is distorted across the sphere, owing to the no-slip boundary conditions. At $R e_{\mathrm{f}}=0.1$ (Fig. 1a), while the fluid approaches the inlet of the channel, a deceleration in fluid velocity occurs. The fluid tends to flow along the pathway with less resistance, and thus part of the fluid flows along the external surface of sphere and the remainder passes through the pore. At $R e_{\mathrm{f}}=40$ (Fig. 1b), owing to the rather strong "advective flow," the boundary layer separation and the after-sphere wake have not occurred. Wu and Lee [32] noted a similar flow pattern behind a uniformly porous sphere. As the porosity is increased to 0.99 (Figs. 1c and 1d), the solids wall only affects the nearby fluid flow owing to surface friction. 


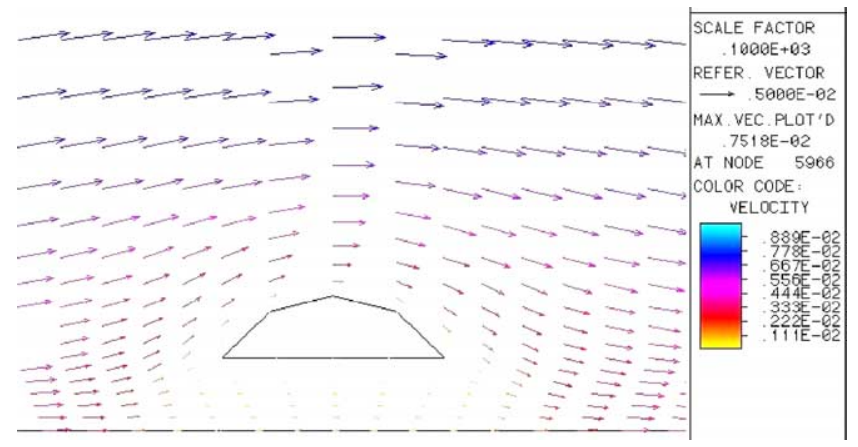

(a)

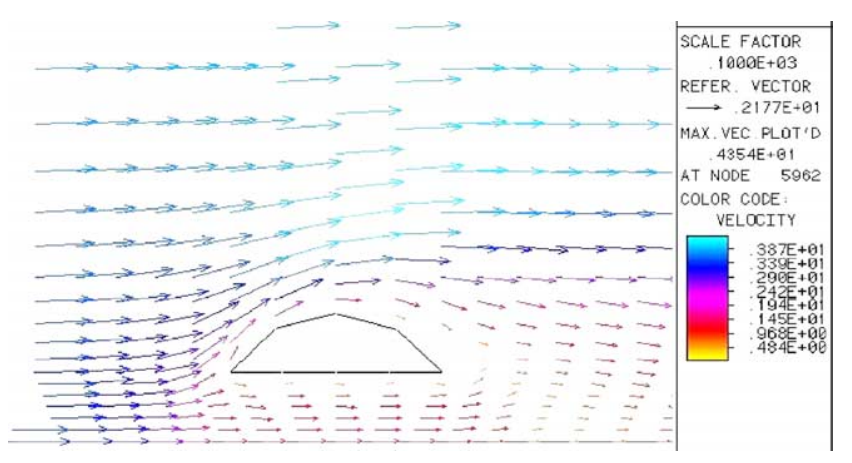

(b)

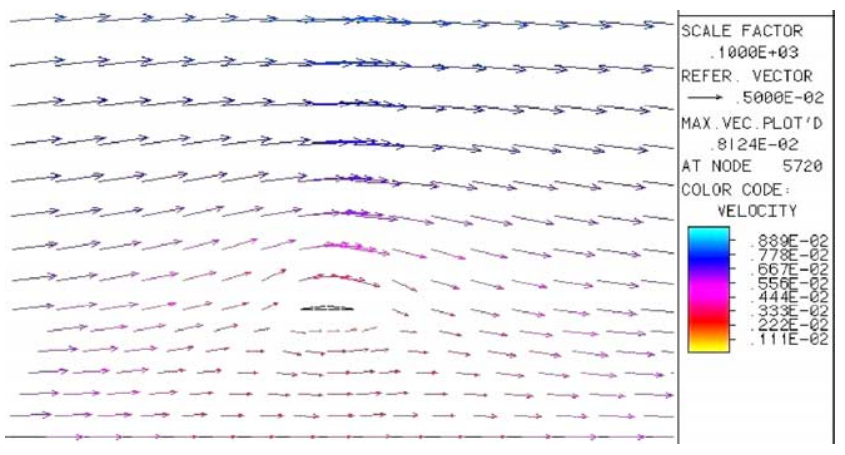

(c)

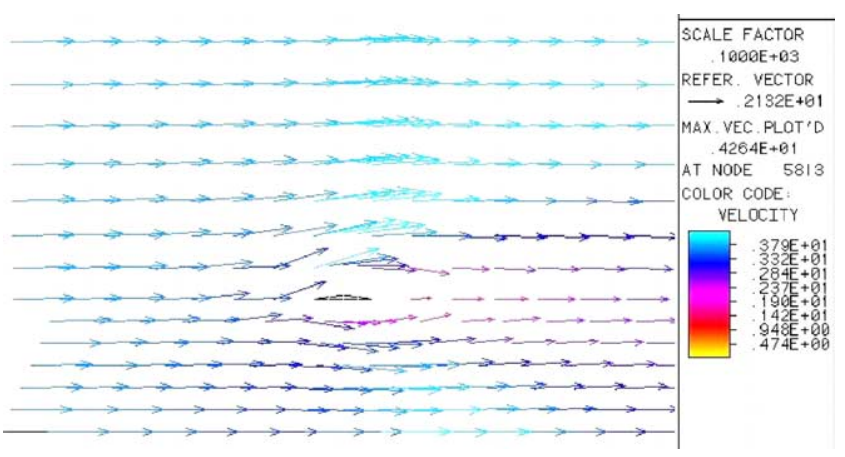

(d)

Fig. 1. Calculated fluid velocity plots (a) $R e_{\mathrm{f}}=0.1, \varepsilon=0.40$; (b) $R e_{\mathrm{f}}=40$, $\varepsilon=0.40$; (c) $R e_{\mathrm{f}}=0.1, \varepsilon=0.99$; (d) $R e_{\mathrm{f}}=40, \varepsilon=0.99$. (Original is in color.)

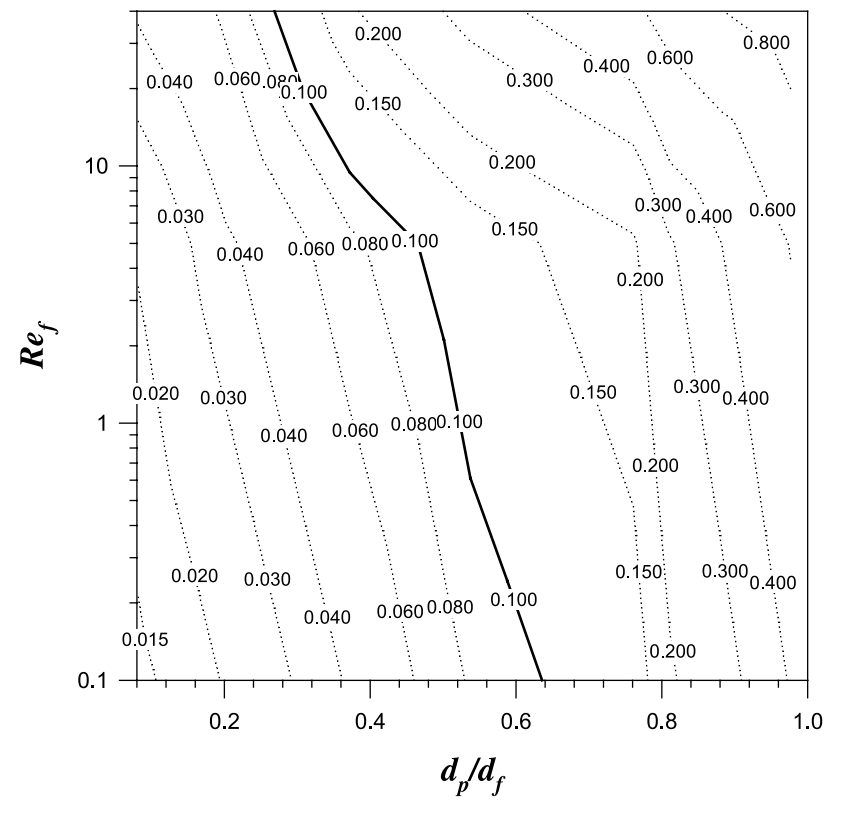

Fig. 2. The $u_{\mathrm{p}, \text { avg }} / U$ as a function of $d_{\mathrm{p}} / d_{\mathrm{f}}$ and $R e_{\mathrm{f}}$.

The average velocity, $u_{\mathrm{p} \text {,avg }}$, at the pore inlet is defined as follows:

$u_{\mathrm{p}, \mathrm{avg}}=\frac{1}{A_{\mathrm{p}}} \int u_{\mathrm{p}} d A_{\mathrm{p}}$.

A significant deceleration of pore velocity to $10 \%$ of the bulk velocity $(U)$ occurs at $d_{\mathrm{p}} / d_{\mathrm{f}}=0.64$ or 0.29 for $R e_{\mathrm{f}}=0.1$ or 40 , respectively (Fig. 2). In the typical range of diameter ratios in activated sludge flocs $(1-20 \%), u_{\mathrm{p}, \mathrm{avg}} / U$ is less than 0.06 , and in most cases less than 0.02 . This result indicates a relatively weak pore flow regardless of the bulk hydrodynamic environment.

\subsubsection{Fluid permeability}

As Eq. (2) stated, the point permeability can be defined as the ratio between $\mu \vec{U}_{\text {rel }}$ and $-\nabla p$, which can be modified for the apparent velocity through the channel of the sludge floc as

$k_{\mathrm{HP}}=-\frac{\mu}{\nabla p} \vec{U}_{\mathrm{rel}}=\frac{\mu}{(-\Delta p / L)}\left(u_{\mathrm{p}, \mathrm{avg}} \frac{d_{\mathrm{p}}}{d_{\mathrm{f}}}\right)^{2}$,

where $L$ is the pore length and $\Delta p$ denotes the pressure drop across the channel pore. The channel flow of the mean velocity of $u_{\mathrm{p} \text {,avg }}$ can be regarded as an equivalent flow field approaching the sphere at a mean velocity $u_{\mathrm{p}, \text { avg }}\left(d_{\mathrm{p}} / d_{\mathrm{f}}\right)^{2}$. For comparison, the permeability based on the Darcy-Brinkman model was calculated as follows. First, the drag force through the floc channel $\left(F_{\text {channel }}\right)$ and that over the external surface $\left(F_{\text {ext }}\right)$ of the mode sphere were evaluated using FIPOST, and hence the total drag force acting on the sphere would be $F=F_{\text {channel }}+F_{\text {ext }}$. As defined in Eq. (1), the correction factor $\Omega$ can be estimated as

$\Omega=\frac{F_{\mathrm{ext}}+F_{\mathrm{int}}}{F_{\mathrm{S}}}$, 


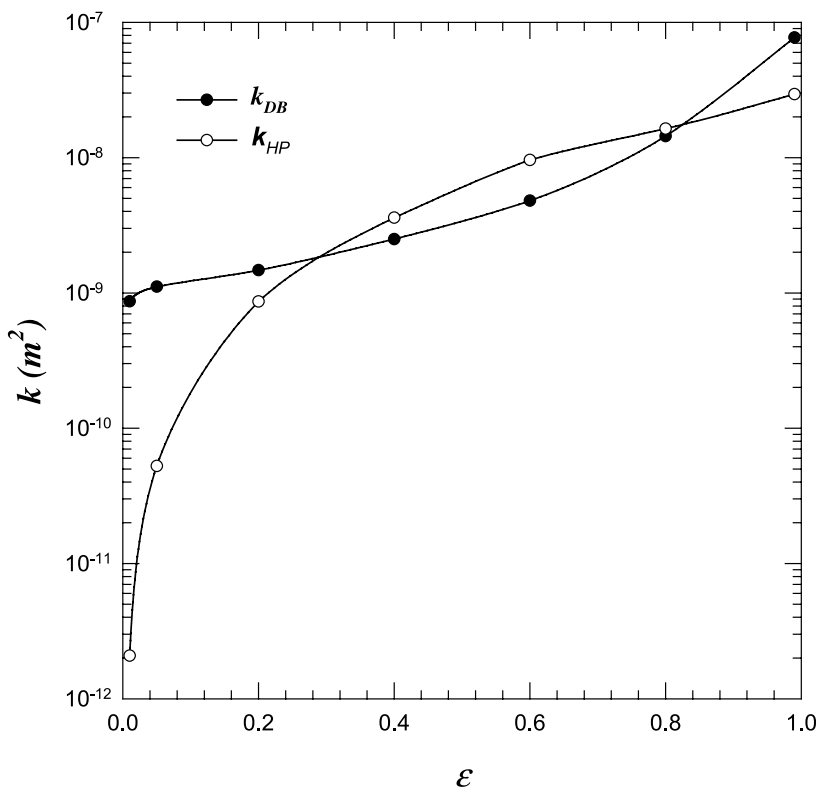

Fig. 3. $k_{\mathrm{HP}}$ and $k_{\mathrm{DB}}$ versus porosity $\varepsilon\left(R e_{\mathrm{f}}=0.1\right)$.

where $F_{\mathrm{S}}$ is the drag force for an impermeable sphere. The corresponding $\beta$ values were obtained based on the $\Omega-\beta$ plots presented by $\mathrm{Wu}$ and Lee [32] (their Fig. 6), assuming a homogeneous sphere interior following the DarcyBrinkman model. The permeabilities thus obtained were denoted as $k_{\mathrm{DB}}$.

The calculated permeabilities for the pored sphere $\left(k_{\mathrm{HP}}\right)$ and those for the homogeneous sphere $\left(k_{\mathrm{DB}}\right)$ are shown in Fig. 3. When the porosity ranges from 0.29 to 0.83 , the homogeneous model underestimates the permeability based on the present floc model, which correlates with the experimental observation [25,33]. Therefore, for a common sludge floc of $d_{\mathrm{p}} / d_{\mathrm{f}}$ less than 0.2 , the homogeneous model overestimates the floc permeability. Particularly for the flocs of low porosity, the former will overestimate the permeability by more than three orders of magnitude. This occurrence could be attributable to the quickly built-up flow resistance across the small pores, as evidenced in Fig. 2 by the low $\left(u_{\mathrm{p} \text {,avg }} / U\right)$ ratio $(<0.02)$ at $d_{\mathrm{p}} / d_{\mathrm{f}}=0.2$ and $R e_{\mathrm{f}}=0.1$. Apparently the configuration of pores, usually difficult to describe, controls the permeability of sludge flocs.

\subsection{Mass transfer}

\subsubsection{Governing equations}

The following Taylor diffusion model controls the dispersive mass transfer process in the sludge pore,

$\frac{\partial c_{\mathrm{A}}^{*}}{\partial t^{*}}+\vec{u}_{\mathrm{p}}^{*} \cdot \nabla^{*} c_{\mathrm{A}}^{*}=\frac{1}{P e} \nabla^{* 2} c_{\mathrm{A}}^{*}$,

where $c_{\mathrm{A}}^{*}=c_{\mathrm{A}} / c_{\mathrm{A}_{0}}, t^{*}=t / t_{0}, P e=d_{\mathrm{f}} u_{\mathrm{p}, \mathrm{avg}} / D_{\mathrm{AW}}, t_{0}$ is

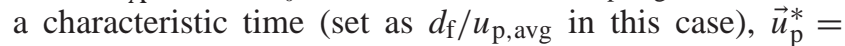
$\vec{u}_{\mathrm{p}} / u_{\mathrm{p} \text {,avg }}$ is the dimensionless pore velocity, and $D_{\mathrm{AW}}$ is the molecular diffusivity of the model component in water. We set $D_{\text {AW }}$ as $5.22 \times 10^{-10} \mathrm{~m}^{2} / \mathrm{s}$ for sucrose in water ([34]; at infinite dilution, $25^{\circ} \mathrm{C}$ ) and $c_{\mathrm{A}_{0}}$ as $1000 \mathrm{mg} / 1$, a concentration typical to wastewater influent. The initial condition and boundary conditions are stated as follows:

$c_{\mathrm{A}}^{*}=0 \quad$ at $t^{*}=0$,

$c_{\mathrm{A}}^{*}=1 \quad$ at $z^{*}=0$ and $t^{*}>0$,

$\nabla^{*} c_{\mathrm{A}}^{*}=0$

or

$c_{\mathrm{A}}^{*}=0$ at sphere's surface,

$\frac{\partial c_{\mathrm{A}}^{*}}{\partial r^{*}}=0 \quad$ at $r^{*}=0$.

Equations (9a) and (9b) state that the substrate concentration is initially set at $c_{\mathrm{A}_{0}}$ ahead of the floc, which flows with the approaching fluid towards the floc. Equation (9c) says that the solids phase is impermeable to mass flow, while Eq. (9d) presents an ultrafast reaction at the sphere surface.

\subsubsection{Concentration profiles with impermeable surface}

The concentration front moves faster along the external surface of the sphere than through the pore (Fig. 4). At a porosity of 0.40 , the subtrate progresses slower through the pore inlet than over the sphere, while the substrate can diffuse from the bulk flow into the pore from its exit side. A local minimum concentration of substrate is noticeable near the middle of the pore (Fig. 4a). In the case of $\varepsilon=0.99$, the concentration profile propagation is merely controlled by the convection flux (Fig. 4b).

Defining the average concentration $c_{\mathrm{A}_{0}}$ over the cross section, with the help of the equation of continuity for an incompressible fluid, Eq. (8) can be rearranged as follows:

$A_{\mathrm{p}} \frac{\partial c_{\mathrm{Aa}}^{*}}{\partial t^{*}}+\iint \nabla \cdot\left(c_{\mathrm{A}}^{*} \vec{u}_{\mathrm{p}}^{*}\right) d A_{\mathrm{p}}-\frac{A_{\mathrm{p}}}{P e_{\mathrm{dis}}} \nabla^{* 2} c_{\mathrm{Aa}}^{*}=0$.

Considering that

$\nabla \cdot\left(c_{\mathrm{A}}^{*} \vec{u}_{\mathrm{p}}^{*}\right)=\nabla \cdot\left(c_{\mathrm{A}}^{*}\left(\vec{u}_{\mathrm{p}}^{*}-\overrightarrow{\left\langle u_{\mathrm{p}}^{*}\right\rangle}\right)\right)+\nabla \cdot\left(c_{\mathrm{A}}^{*} \overrightarrow{\left\langle u_{\mathrm{p}}^{*}\right\rangle}\right)$,

where $\left\langle u_{\mathrm{p}}^{*}\right\rangle$ is the mean velocity of pore fluid, Eq. (10) can be further rearranged into

$\frac{\partial c_{\mathrm{Aa}}^{*}}{\partial t^{*}}-\frac{1}{P e_{\text {tot }}} \nabla^{* 2} c_{\mathrm{Aa}}^{*}+\nabla \cdot\left(c_{\mathrm{Aa}}^{*} \overrightarrow{\left\langle u_{\mathrm{p}}^{*}\right\rangle}\right)=0$,

where the following relation was assumed: $\iint c_{\mathrm{A}}^{*}\left(\vec{u}_{p}^{*}-\right.$ $\left.\overrightarrow{\left\langle u_{\mathrm{p}}^{*}\right\rangle}\right) d A_{\mathrm{p}}=\left(-A_{\mathrm{p}} / P e_{\mathrm{dis}}\right) \nabla c_{\mathrm{Aa}}^{*}$, with $P e_{\mathrm{dis}}=d_{\mathrm{f}} u_{\mathrm{p}, \mathrm{avg}} / E_{\mathrm{ax}}$; $\overrightarrow{\left\langle u_{\mathrm{p}}^{*}\right\rangle}$ is the vector $(0,0,1)$ in the present case; $E_{\mathrm{ax}}$ is the axial dispersion coefficient; and $P e_{\text {tot }}=d_{\mathrm{f}} u_{\mathrm{p}, \text { avg }} /\left(E_{\mathrm{ax}}+D_{\mathrm{AW}}\right)$. Integrating Eq. (11) over the pore length yields the mass balance equation along the pore axial direction as follows:

$\frac{\partial \int c_{\mathrm{Aa}}^{*} d z^{*}}{\partial t^{*}}+\left.c_{\mathrm{Aa}}^{*}\right|_{z^{*}=1}=\left.\frac{1}{P e_{\mathrm{tot}}} \frac{\partial c_{\mathrm{Aa}}^{*}}{\partial z^{*}}\right|_{z=1}$.

Restated, $P e_{\text {tot }}$ can be evaluated by finding the slope of the (total mass flow rate-convective mass flow rate) versus 


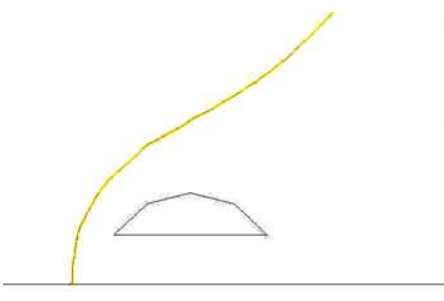

(a) $t^{*}=720$

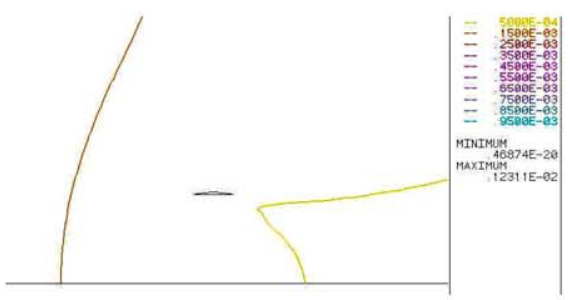

(d) $t^{*}=720$

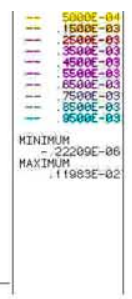

1

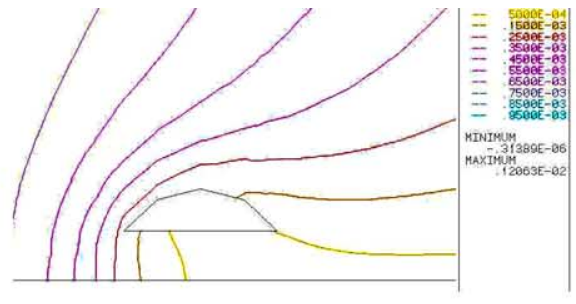

(b) $t^{*}=780$

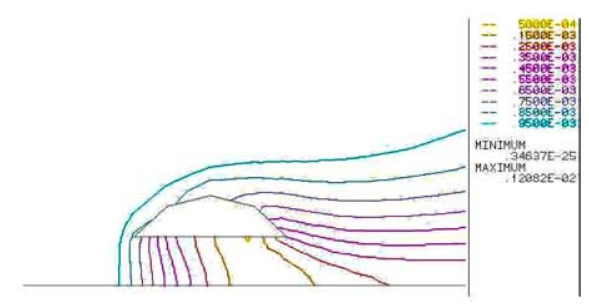

(c) $t^{*}=840$

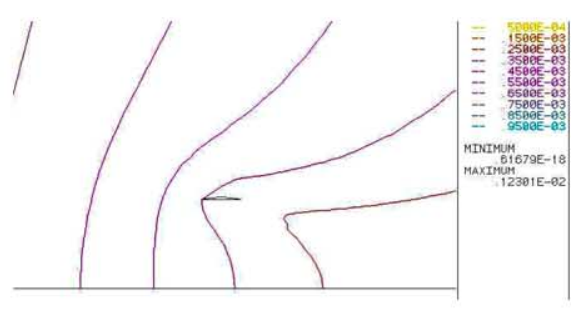

(e) $t^{*}=780$

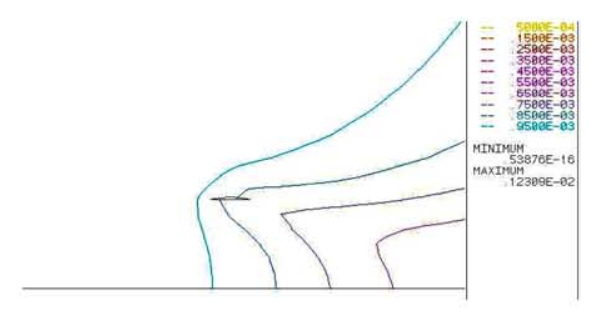

(f) $t^{*}=840$

Fig. 4. Propagation of the substrate concentration profiles at $\operatorname{Re}_{\mathrm{f}}=0.1$ : $(\mathrm{a}-\mathrm{c}) \varepsilon=0.40$, $(\mathrm{d}-\mathrm{f}) \varepsilon=0.99$. (Original is in color.)

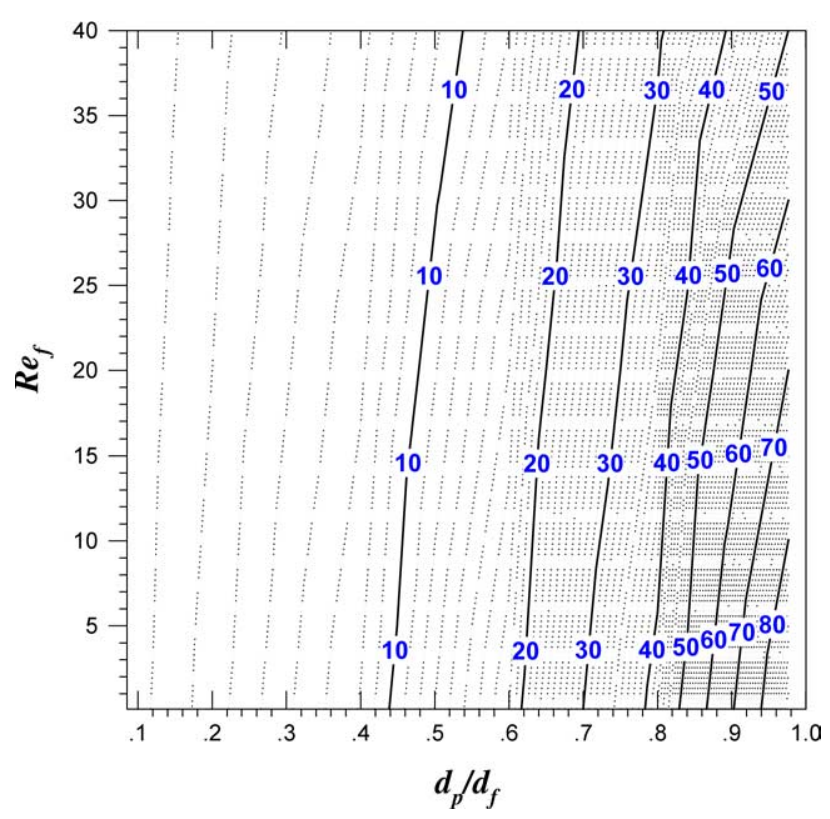

Fig. 5. The ratio of dispersion coefficient $E_{\text {tot }}$ and the molecular diffusivity $D_{\text {AW }}$ of straight pores.

the concentration gradient at the exit, based on which the total dispersion coefficient $E_{\mathrm{tot}}=E_{\mathrm{ax}}+D_{\mathrm{AW}}$ can be obtained.

The coefficient ratio, $E_{\text {tot }} / D_{\mathrm{AW}}$ increases dramatically with the porosity and the Reynolds number (Fig. 5) for the straight pore in the model floc (Fig. 1). At $d_{\mathrm{p}} / d_{\mathrm{f}}>0.12$, the dispersive transport can be two times the molecular diffusion. For instance, at $R e_{\mathrm{f}}=0.1, E_{\text {tot }}=6.8 \times 10^{-10}$ or $9.4 \times 10^{-9} \mathrm{~m}^{2} / \mathrm{s}$ at $d_{\mathrm{p}} / d_{\mathrm{f}}=0.1$ or 0.6 , respectively. This enhancement of the mass transport is not attributable to the convective flux $\left(c_{\mathrm{A}_{0}} u_{\mathrm{p} \text {,avg }}\right)$, but is contributed by the Taylor dispersion effect. Both convection and diffusion of mass are significant in the intrafloc transport process.

\subsubsection{Zero surface concentration}

The above section examines the case with an impermeable wall, which is an extreme condition assuming no interfacial mass transfer. The substrate is absorbed and penetrated into the surface of a real floc. Normally this interfacial transfer rate is slow, controlled by the microbial activity within the floc. Another extreme condition indicates an extremely rapid reaction to occur at the floc surface, thereby producing a nearly zero substrate concentration (Eq. (9d)). In this extreme, a rather steep concentration gradient is developed at the near-surface regime, indicating a strong interfacial mass flux. The $E_{\text {tot }}$ thus calculated for $\varepsilon=0.4$ at $R e_{\mathrm{f}}=0.1\left(3.1 \times 10^{-9} \mathrm{~m}^{2} / \mathrm{s}\right)$ is approximately $60 \%$ lower than the value from Fig. $4\left(7.8 \times 10^{-9} \mathrm{~m}^{2} / \mathrm{s}\right)$. For most sludge flocs, the microbial activity is not sufficiently high to induce a strong interfacial mass flux. Hence, the cases demonstrated in Fig. 4 should present the real process better than the zero-concentration limit. Nonetheless, the present example reveals that the possible error to assume an impermeable surface is much less than $60 \%$.

In the next section we numerically simulate the flow field and mass transfer characteristics for a single pore in a real floc.

\section{Flow in floc pore}

\subsection{Experimental}

Waste-activated sludge was sampled from the wastewater treatment facility at the Neili Bread Plant, Presidential Enterprise Co., Taoyuan, Taiwan. The total solid content (TS) was measured as $9350 \mathrm{mg} / 1$. An Accupyc 1330 pycnometer (Micromeritics, UK) measured the density of the dried solid $\left(\rho_{\mathrm{S}}\right)$ in sludge at $1450 \mathrm{~kg} / \mathrm{m}^{3}$, with a relative deviation of less than $0.5 \%$. The $\mathrm{pH}$ value of the original sludge was around 7.0 . 


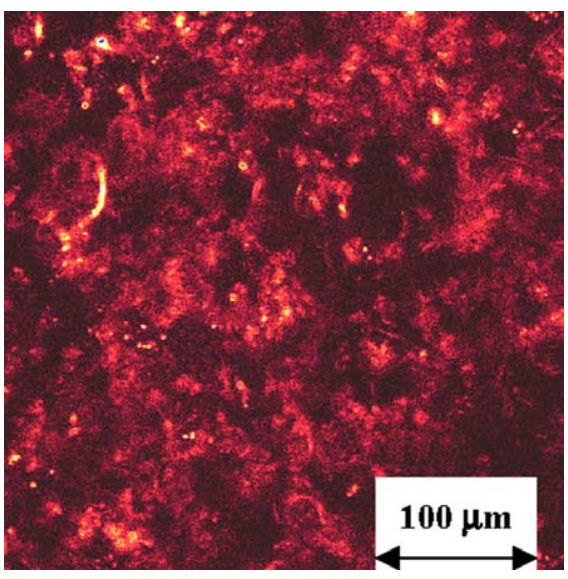

(a)

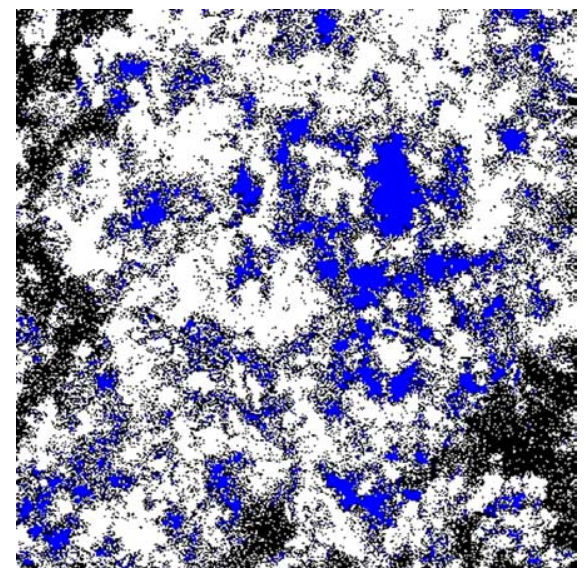

(b)

Fig. 6. Demonstration of the CLSM image and the blob analysis results: (a) CLSM image; (b) blob analysis (all blobs). (Original is in color.)

A confocal laser scanning microscope (CLSM; Leica TCS SP2, Germany) was used to observe the internal floc structure. This microscope was equipped with image processing software and an argon laser source was used to stimulate fluorescence. The sludge floc of diameter $328 \mu \mathrm{m}$ was carefully sampled and imaged using $20 \times, 40 \times$, or $60 \times$ objectives, depending on the required resolution. The microscope scanned the samples at a fixed depth and the obtained image was digitized. Sludge samples were first chemically fixed and then embedded in agarose to perform fluorescent in situ hybridization (FISH). This study used DNA probes Eub 338 (labeled by rhodamine) to detect most eubacteria in biosolids. Their nucleotide sequence is 5'-GCTGCCTCCCGTAGGAGT-3'.

After the probe was added, hybridization was performed at $50^{\circ} \mathrm{C}$ for $1 \mathrm{~h}$. The stained samples were washed three times under the same conditions, using hybridization buffer solution to remove extra probes. CLSM scanned every slice three times and then averaged the image to remove the luminescent noise. Sliced images in RGB (red-green-blue) mode were first converted to grayscale images to construct a histogram of pixels versus luminescence intensity by IN-

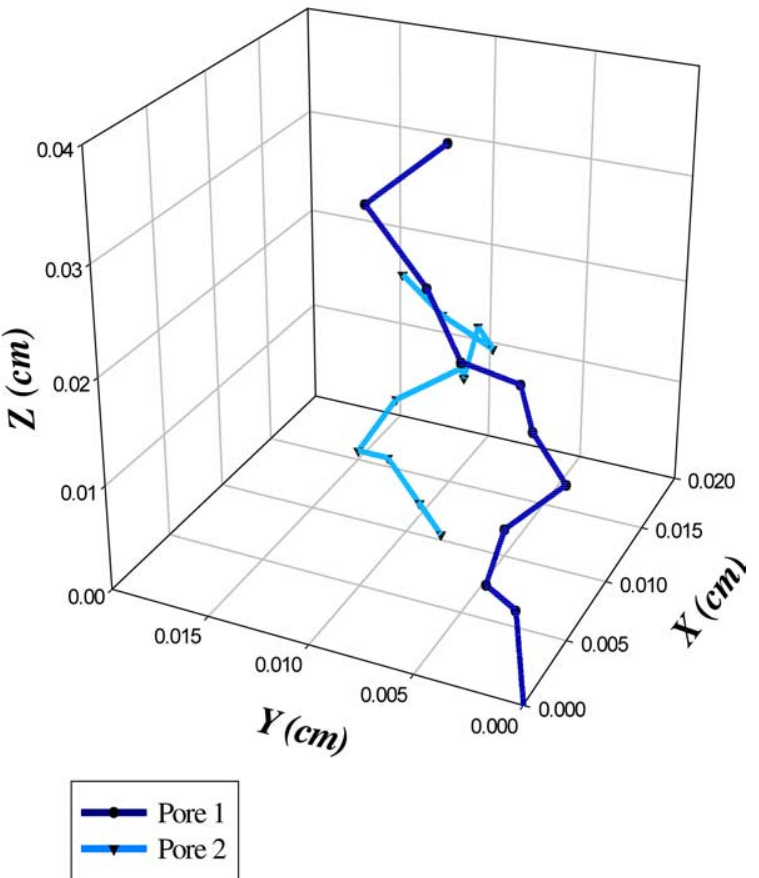

(a)

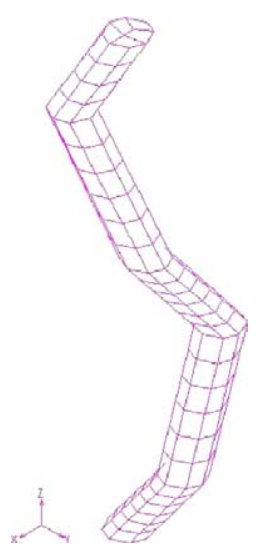

(b)

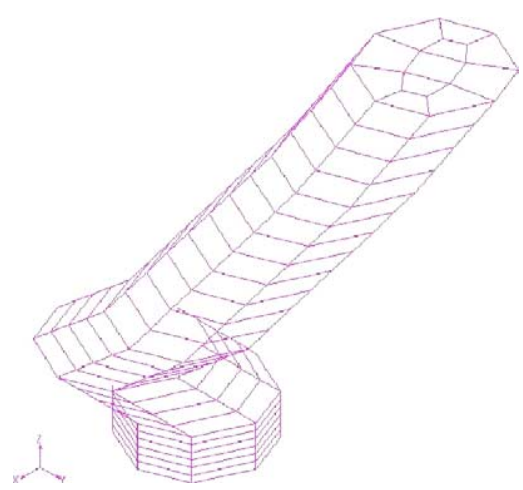

(c)
Fig. 7. Configuration of the two largest pores in the sludge floc. (a) Space configuration of the two pores; (b) computational domain for pore 1 : $d_{\mathrm{p}}=27.6 \mu \mathrm{m} ; d_{\mathrm{p}} / d_{\mathrm{f}}=0.084 ; \tau=1.24$; (c) computational domain for pore $2: d_{\mathrm{p}, \mathrm{avg}}=33.3 \mu \mathrm{m} ; d_{\mathrm{p}} / d_{\mathrm{f}}=0.102 ; \tau=1.55$.

SPECTOR (Matrox, Canada). A region of interest (ROI) was defined on the microtome-sliced image to include sufficient morphological detail for analysis and to avoid the inhomogeneity of the luminescent background [35].

\subsection{Model and solutions}

The geometry of the tortuous pores is obtained from the slices of CLSM images. First a series of continuous scanning images on one floc is selected. A thresholding value is set to separate the background (the dark portion in CLSM images) and foreground (the biomass in the floc, the white portion) for all images of this series. The original CLSM image is demonstrated in Fig. 6a. The blob analysis is then carried out 


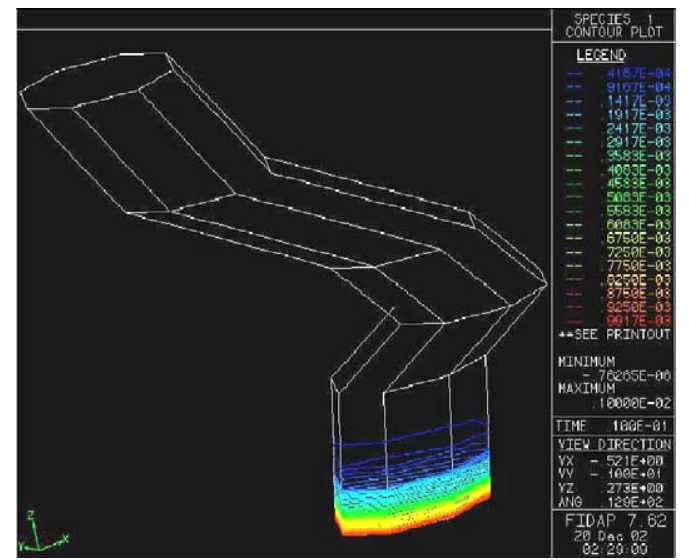

(a)

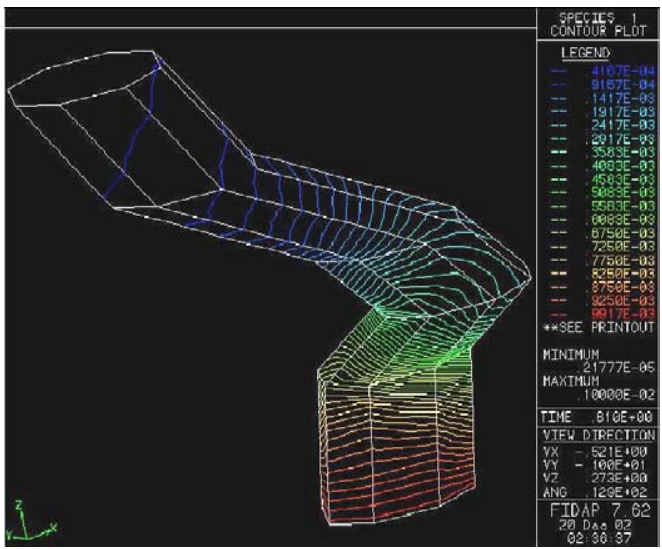

(c)

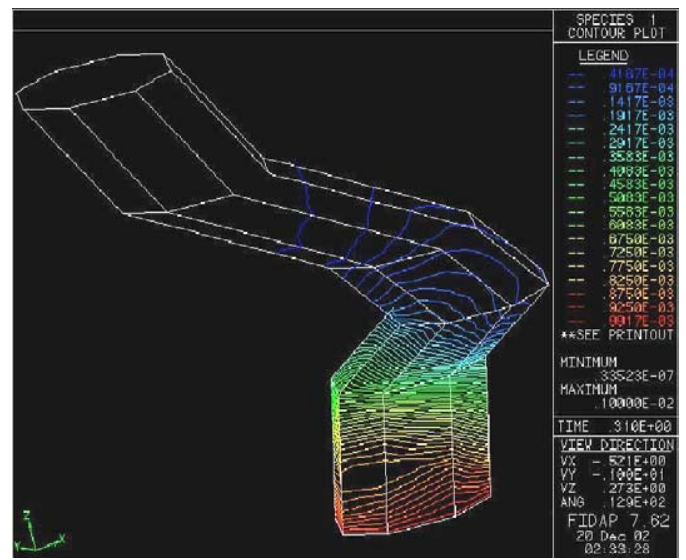

(b)

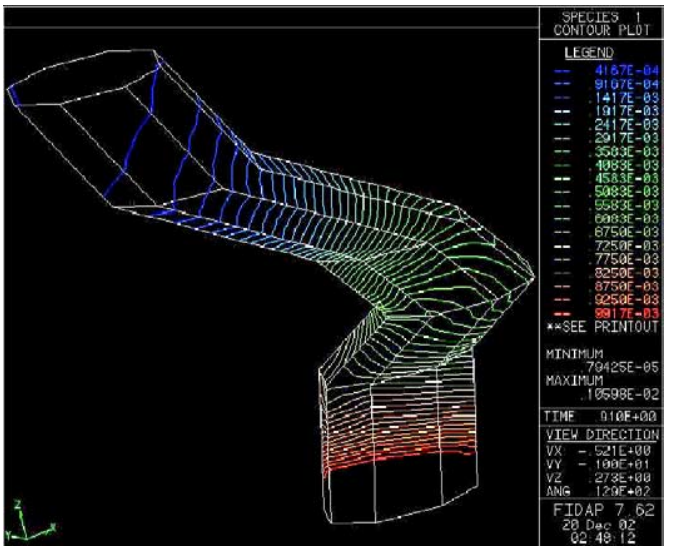

(d)

Fig. 8. Evolution of concentration profiles for pore 2 . $R e_{\mathrm{f}}=0.1 ; L=220 \mu \mathrm{m}$. (a) Initially the solute A was placed at the pore inlet; (b) $t^{*}=50$; (c) $t^{*}=100$; (d) $t^{*}=150$. (Original is in color.)

to position the pores in the floc. The dark portions touching the image boundary are excluded since they are not cavities involved in the floc biomass. There are 2657 blobs detected in Fig. 6b, but only 40 of them are big pores of diameter exceeding $10 \mu \mathrm{m}$. Most large pores are not interconnected. Hence, the flow process through each pore can be regarded as free of interruption from others. The effects of the smaller pores beside the big pore are discussed further later.

Two largest pores are identified whose space configurations are shown in Fig. 7a. The real pores are tortuous in space and in some cases the pores run outside and then connect back to the floc several $\mu \mathrm{m}$ away. Here we take a typical section from both pores with both ends open to the surrounding fluid as sample calculations (Figs. 7b and 7c). For pore 1, the average pore diameter and the tortuosity factor $(\tau)$ are 27.6 and $1.24 \mu \mathrm{m}$, respectively. For pore 2, the corresponding values are 33.3 and $1.55 \mu \mathrm{m}$, respectively. Over the 40 pores identified from the floc, the tortuosity factor ranges from 1.03 to 2.5 .

The governing equations controlling the fluid flow and the mass transfer of solute in the pores are Eqs. (3) and (7) in their 3D forms, which are numerically solved using FIDAP 7.60 together with the boundary conditions, Eqs. (4a)-(4c), (9a)-(9c), and (9e). The length $L$ here denotes the real length of the tortuous pore, while at $t^{*}=0$ the solute concentration at inlet is set at $c_{\mathrm{A}}=c_{\mathrm{A}_{0}}$ at $t=0$. Ten pores are selected to cover the tortuosity factor range of interest. The average pore velocity for each pore is estimated using data presented in Fig. 2.

The evolution of solute in pore 2 is shown in Fig. 8. Solutes in the pore have moved along a curved line. At each cross section the solute is not evenly distributed since the change in flow direction at each turn leads to an enrichment of solute at the near end of the turn. Pore tortuosity definitely has a significant effect on the flow process in the sludge pores.

The permeability and the dispersion coefficients can be estimated for each pore investigated using Eqs. (6) and (10). For example, the permeability and dispersion coefficient of the pore sketched in Fig. 7c are $2.12 \times 10^{-12} \mathrm{~m}^{2}$ and $5.8 \times 10^{-9} \mathrm{~m}^{2} / \mathrm{s}$ at $R e_{\mathrm{f}}=0.1$, respectively. The corresponding values for straight pores of identical pore diameter and pore length are $4.8 \times 10^{-11} \mathrm{~m}^{2}$ and $6.8 \times 10^{-8} \mathrm{~m}^{2} / \mathrm{s}$, one order of magnitude larger than the floc pore results. At a higher Reynolds number of 40, this effect has become slightly more significant, owing to the considerable reduction in inflow ve- 

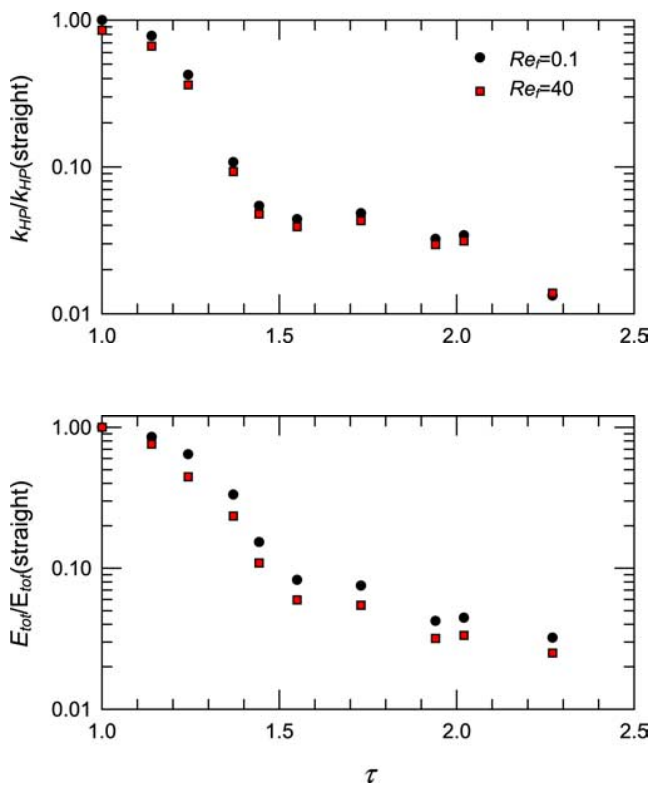

Fig. 9. The ratio of permeability and dispersion coefficient between the floc pore and the straight pore.

locity after entering the pore (Fig. 9). Although the pores under investigation are of various lengths and shapes, apparently the tortuous structure of the pore considerably retards the flow field and convective mass transfer. Hence, beside the Taylor dispersion effect, pore tortuosity controls the intrafloc transport process. Results based on a floc model without detailed information on interior structure would be misleading. A single index such as porosity is not sufficient to predict process performance involving flocs.

In CLSM images some "side pores" can be identified to connect to the large pores under consideration. A sample calculation considers the solute transport (diffusion and convection) across a junction at which a pore $\left(d_{\mathrm{p}}=33 \mu \mathrm{m}\right)$ is split into one side pore $(28 \mu \mathrm{m})$ and another much smaller pore $(5 \mu \mathrm{m})$ (Fig. 10). It is clear that the convective transport to the side pore is much less significant than that to the mother pore. Hence, as mentioned above, we can neglect the role of side pores in the flow process in the main pores considered.

\section{Conclusions}

The present work revealed the intrafloc transport processes, considering the fluid flow and mass transport in pores identified for activated sludge floc. Simulation of a sphere with one straight pore passing through its center revealed that although large pores control the advective flow through a sludge floc, if the pore size were lower than $20 \%$ of the floc size, the homogeneous model would overestimate the permeability based on the present floc model. Meanwhile, the pore velocity would be less than $2 \%$ of the bulk velocity, indicating a relatively weak pore flow regardless of the bulk

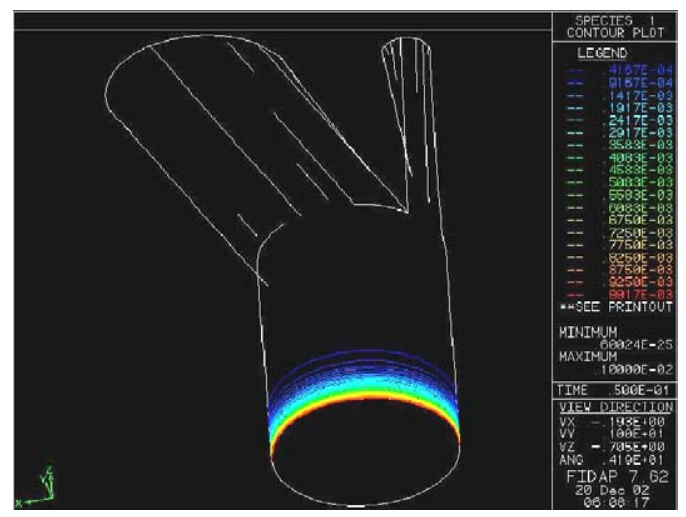

(a)

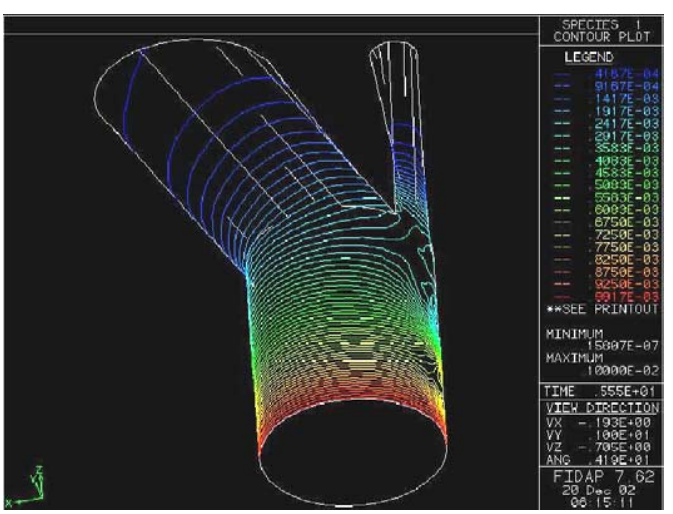

(b)

Fig. 10. Evolution of concentration profiles for a split pore. Mother pore: $d_{\mathrm{p}}=33 \mu \mathrm{m}$; daughter pores: $d_{\mathrm{p}}=28$ and $5 \mu \mathrm{m}$, respectively. (a) Initially the solute A was placed at the inlet of the mother pore; (b) $t^{*}=50$. (Original is in color.)

hydrodynamic environment. For instance, at $R e_{\mathrm{f}}=40$, the corresponding pore Reynolds number based on pore diameter and pore velocity would be less than 0.4 . This relatively low advective flow indicates a relative insignificant convective transport within sludge floc. On the other hand, although the transport flux $\left(c_{\mathrm{Aa}} u_{\mathrm{p}, \mathrm{avg}}\right)$ is not as significant as imaged, the convective flow facilitates the mass diffusion rate contributed by the uneven distribution of the pore velocity over the cross section.

Real pores in activated sludge floc were observed using fluorescent in situ hybridization (FISH) followed by confocal laser scanning microscope (CLSM) imaging. Threedimensional geometry of pores of size exceeding $10 \mu \mathrm{m}$ was recorded and the numerical model was established. Over the 40 pores identified from the floc imagery, the tortuosity factor ranges from 1.03 to 2.5 . Within sludge pores, besides the Taylor dispersion effect, the tortuous structure of the pore considerably retards the flow field and dispersive mass transfer. Moreover, the role of "side pores" appears to be minor in comparison with the mainstream transport process. Nonetheless, a comprehensive floc model should incorporate information on the interior structure to detailing the intrafloc transport processes. 


\section{References}

[1] D.J. Lee, G.W. Chen, Y.C. Liao, C.C. Hsieh, Water Res. 30 (1996) 541.

[2] R.M. Wu, W.H. Feng, I.H. Tsai, D.J. Lee, Water Environ. Res. 70 (1998) 1258.

[3] R.M. Wu, G.W. Tsou, D.J. Lee, Adv. Environ. Res. 4 (2000) 163.

[4] R.M. Wu, G.W. Tsou, D.J. Chem. Eng. J. 80 (2000) 37.

[5] G.W. Tsou, R.M. Wu, P.S. Yen, D.J. Lee, X.F. Peng, J. Colloid Interface Sci. 250 (2002) 400.

[6] X.Y. Li, Y.A. Yuan, Water Res. 36 (2002) 3110.

[7] X.Y. Li, Y. Yuan, H.W. Wang, Environ. Sci. Technol. 37 (2003) 292.

[8] P. Tang, J.A. Raper, Powder Technol. 123 (2002) 114.

[9] G.C. Bushell, Y.D. Yan, D. Woodfield, J. Raper, R. Amal, Adv. Colloid Interface Sci. 95 (2002) 1.

[10] B.E. Logan, J.R. Hunt, Limnol. Oceanogr. 32 (1987) 1034.

[11] B.E. Logan, J.R. Hunt, Biotechnol. Bioeng. 31 (1988) 91.

[12] F. Zartarian, C. Mustin, G. Villemin, T. Ait-Ettager, A. Thill, J.Y. Bottero, J.L. Mallet, D. Snidaro, Langmuir 13 (1997) 35.

[13] A. Thill, S. Veerapaneni, B. Simon, M.R. Wiesner, J.Y. Bottero, D. Snidaro, J. Colloid Interface Sci. 204 (1998) 357.

[14] R.M. Wu, D.J. Lee, T.D. Waite, J. Guan, J. Colloid Interface Sci. 252 (2002) 383.
[15] H.Y. Chung, D.J. Lee, J. Colloid Interface Sci. 267 (2003) 136.

[16] H.Y. Chung, S.P. Ju, D.J. Lee, J. Colloid Interface Sci. 263 (2003) 498.

[17] D.J. Lee, Water Res. 33 (1999) 1116.

[18] K. Matsumoto, A. Suganuma, D. Kunii, Chem. Eng. Sci. 33 (1978) 1554.

[19] D.H. Li, J. Ganczarczyk, Water Environ. Res. 64 (1992) 236.

[20] H. Huang, Clay Clay Miner. 41 (1993) 373.

[21] S.N. Rogak, R.C. Flagan, J. Colloid Interface Sci. 134 (1990) 206.

[22] S. Chellam, M.R. Wiesner, Water Res. 27 (1993) 1943.

[23] X.Y. Li, B.E. Logan, Environ. Sci. Technol. 31 (1997) 1229.

[24] X.Y. Li, B.E. Logan, Environ Sci. Technol. 31 (1997) 1237-1242.

[25] X.Y. Li, B.E. Logan, Water Res. 35 (2001) 3373.

[26] S. Veerapaneni, M.R. Wiesner, J. Colloid Interface Sci. 177 (1996) 45.

[27] R.M. Wu, D.J. Lee, P.J. He, J. Chin. Inst. Chem. Eng. 34 (2003) 275.

[28] S. MacIntyre, A.L. Alldredge, C.C. Gotschalk, Limnol. Oceanogr. 39 (1995) 449.

[29] A.L. Alldredge, K.M. Crocker, Sci. Total Environ. 165 (1995) 15.

[30] H. Ploug, Limnol. Oceanogr. 46 (2001) 1624.

[31] D. Woodfield, G. Bickert, Water Res. 16 (2001) 3801.

[32] R.M. Wu, D.J. Lee, Water Res. 32 (1998) 760.

[33] M.R. Wiesner, J. Environ. Eng. 125 (1999) 1124.

[34] E.L. Cussler, Diffusion Mass Transfer in Fluid Systems, second ed., Cambridge Univ. Press, New York, 1997.

[35] C.P. Chu, D.J. Lee, Environ. Sci. Technol. 38 (2004) 1161. 